\title{
Métabolomique, métabonomique et les préoccupations en matière de sécurité alimentaire et de protection de la santé des consommateurs*
}

\author{
Alain PARIS ${ }^{1}$ \\ Céline DOMANGE ${ }^{1}$ \\ Alexandre EVEILLARD ${ }^{2}$ \\ Cécile CANLET ${ }^{1}$ \\ Pascal MARTIN ${ }^{2}$ \\ Thierry PINEAU ${ }^{2}$ \\ Nathalie PRIYMENKO ${ }^{1}$ \\ ${ }^{1}$ UMR1089 - Xénobiotiques, INRA-ENVT, \\ Toulouse \\ <aparis@toulouse.inra.fr> \\ ${ }^{2}$ UR66 - Pharmacologie-Toxicologie, INRA, \\ Toulouse
}

La métabonomique conjugue deux approches, la première, la métabolomique, démarche analytique qui consiste à produire à haut débit et à quantifier des empreintes métaboliques, la seconde, la statistique multivariée, qui consiste à traiter les données pour faire apparaître les facteurs structurants qui permettent de résumer l'information, eu égard au questionnement à l'origine de l'exploration fonctionnelle ainsi réalisée. Le terme «métabonomique » a été forgé par Nicholson et ses collègues de I'Imperial College à l'Université de Londres à la fin des années 90 [1] et s'inscrit dans le courant des méthodes dédiées à la connaissance des organismes, que celles-ci considèrent l'ensemble des ARN transcrits dans la cellule, les protéines qui résultent de leur traduction ou les produits issus des activités métaboliques des enzymes ainsi synthétisées dans la cellule. Toutes ces méthodes partagent en commun : premièrement le fait de générer des quantités importantes de données qui doivent nécessairement être traitées par des outils statistiques adaptés et deuxièmement de s'inscrire dans une démarche « relative » en cela que les biomarqueurs d'intérêt résultent toujours d'une démarche de comparaison des groupes d'individus étudiés dans un contexte de perturbation du fonctionnement «normal » des individus.

\footnotetext{
${ }^{*}$ Conférence issue de la séance du 18 juin 2008 de I'Académie d'Agriculture. Voir la présentation à I'adresse suivante :

www.academie-agriculture.fr/detail-seance_37.html.
}

\begin{abstract}
Metabonomics is a recent method devoted to the high throughput metabolic phenotyping of biological systems usable to perform a deep functional exploration when physiological, toxicological, genetic or nutritional perturbations are considered. From some pivotal examples, will be illustrated some concepts such as "metabolic disruptions" or "set of metabolic biomarkers", which are routinely used in toxicological studies of living systems with the final aim to (i) better characterize some homeostatic rules involved in response to toxic insults and (ii) to better know prediction rules usable to classify unknown individuals given the knowledge of dedicated reference data set. Hereafter are presented illustrative cases concerning the use of steroidal anabolics in growing cattle, the characterization of secondary metabolites present in a plant which is toxic for equine species, or the description of some metabolic effects induced by an endocrine disruptor widely used in plastic industry.
\end{abstract}

Key words: metabonomics, food safety, metabolic disruptions

De cette approche métabonomique peuvent ressortir différents ensembles de biomarquage qui représentent autant d'hypothèses pouvant aider à interpréter les disruptions métaboliques induites par des perturbations toxiques mais aussi à les hiérarchiser. Cet outil possède des propriétés qui s'apparentent de fait à celles d'un microscope, un microscope particulier dit à « réseau métabolique », puisque ce sont les variations dans ce réseau qui permettent de mesurer l'effet des perturbations qui y sont appliquées.

Les tout premiers travaux en métabonomique sont ceux produits par l'équipe de J. Nicholson dans un contexte d'étude pharmacologique ou toxicologique qui ont permis de poser les fondements de l'approche [2, 3]. Depuis, des progrès substantiels dans l'appréhension des effets toxiques de substances, déjà bien décrits par le passé, ont pu être obtenus grâce aux travaux réalisés par ce même groupe $[4,5]$. Ceci a d'ailleurs eu pour répercussion de faire évoluer en profondeur l'évaluation pharmacologique et toxicologique des nouveaux médicaments grâce à un partenariat avec les firmes pharmaceutiques tel qu'il est relaté dans des travaux du consortium COMET [6-8]. Vraisemblablement, ces données à « large spectre » permettront à terme de consolider l'évaluation toxicologique de ces nouvelles substances auprès des agences réglementaires telles I'EMEA en Europe ou la FDA aux Etats-Unis et de mieux garantir l'absence d'effets secondaires, mais aussi corolairement de mieux caractériser à un stade pré- coce de développement les différents médicaments candidats pour éventuellement les retirer ou les repositionner dans un autre champ de prescriptions potentielles eu égard aux nouvelles propriétés pharmacologiques ainsi révélées.

Des travaux que nous avons conduits récemment ont aussi montré que des disruptions métaboliques d'origine hormonale pouvaient être caractérisées par cette approche dans le cas d'animaux traités avec des substances anabolisantes. De même, des variations du métabolisme général liées à l'administration de faibles et fortes doses de phtalates, composés issus de la plasturgie doués de propriétés de disruption endocrinienne pouvaient être mises en regard de celles mesurées en parallèle dans I'expression des ARN messagers. Enfin, I'outil métabonomique a permis de caractériser les cibles d'intoxication d'une plante, réputée neurotoxique pour l'espèce équine, tout en en caractérisant le potentiel toxique dans différents stades végétatifs. C'est au travers de ces différents exemples que les différents atouts de la métabonomique seront décrits dans le domaine de l'évaluation de la sécurité alimentaire du consommateur.

\section{Physiologie et métabonomique : le cas des stéroïdes anabolisants}

À l'opposé des perturbations profondes du fonctionnement du métabolisme général indui- 
tes par l'administration de toxique à des doses supra physiologiques ou pharmacologiques, celles résultant d'une administration contrôlée d'hormones ayant un rôle dans le contrôle de la croissance des animaux sont nécessairement de moindre ampleur et la recherche de potentiels biomarqueurs de l'effet de ces hormones exogènes pouvait rester vaine puisque la mesure des variations du métabolisme général imputables à ces disruptions d'origine endocrinienne n'avait jamais été prouvée préalablement. Pourtant, les données produites en situation expérimentale contrôlée sur bovins mâles et femelles ont permis de révéler des variations métaboliques coordonnées qui pouvaient être expliquées d'abord par un effet majeur lié au genre, puis par un effet de moindre ampleur qui correspondait à une réponse anabolique commune entre mâles et femelles traités aux anabolisants (figure 1), la variation de cette réponse liée spécifiquement au sexe des animaux traités étant mineure [9]. La caractérisation structurale de ces données produites par RMN à haut champ a pu être partiellement réalisée et les biomarqueurs métaboliques ainsi identifiés ont pu être validés au plan biochimique et physiologique d'après les observations éparses publiées sur près de 40 ans [10]. Ainsi, le traitement par une association de type androgèneœstrogène chez les mâles ou par un androgène seul chez les femelles a des répercussions sur le turnover des composés azotés, une moindre excrétion en urée, en créatinine et en créatine étant constatée chez tous ces animaux. Néanmoins, un bypass dans l'excrétion azotée via l'excrétion d'hippurate, un produit de conjugaison du benzoate, lui-même produit de dégradation des noyaux aromatiques, à la glycine existe qui compense très partiellement cette réduction d'excrétion de composés azotés. Concernant le métabolisme énergétique, I'utilisation des anabolisants se traduit dans l'espèce bovine par une augmentation relative de citrate mesuré dans l'urine et une diminution du glucose urinaire. Vraisemblablement, un ajustement coordonné des activités enzymatiques impliquant la lactate déshydrogénase, la pyruvate déshydrogénase et l'isocitrate déshydrogénase pourrait expliquer cette variation du profil urinaire en glucose et en dérivés du cycle tricarboxylique et orienter les réponses attendues en certains dérivés qui n'avaient alors pu être mesurés [10]. L'identification des biomarqueurs et l'interprétation des modifications des voies métaboliques concernées ont ainsi permis de faire émerger une perspective fonctionnelle unifiée qui résume près de 40 ans d'observations biochimiques fractionnaires. Ce travail préliminaire conduit chez l'animal de rente a permis d'initier une étude pilote des disruptions métaboliques induites par une modification des profils endocriniens observée chez les sportifs de haut niveau qui sont soumis depuis quelques années au suivi médical longitudinal organisée avec l'appui des fédérations nationales. II est vraisemblable que des varia-

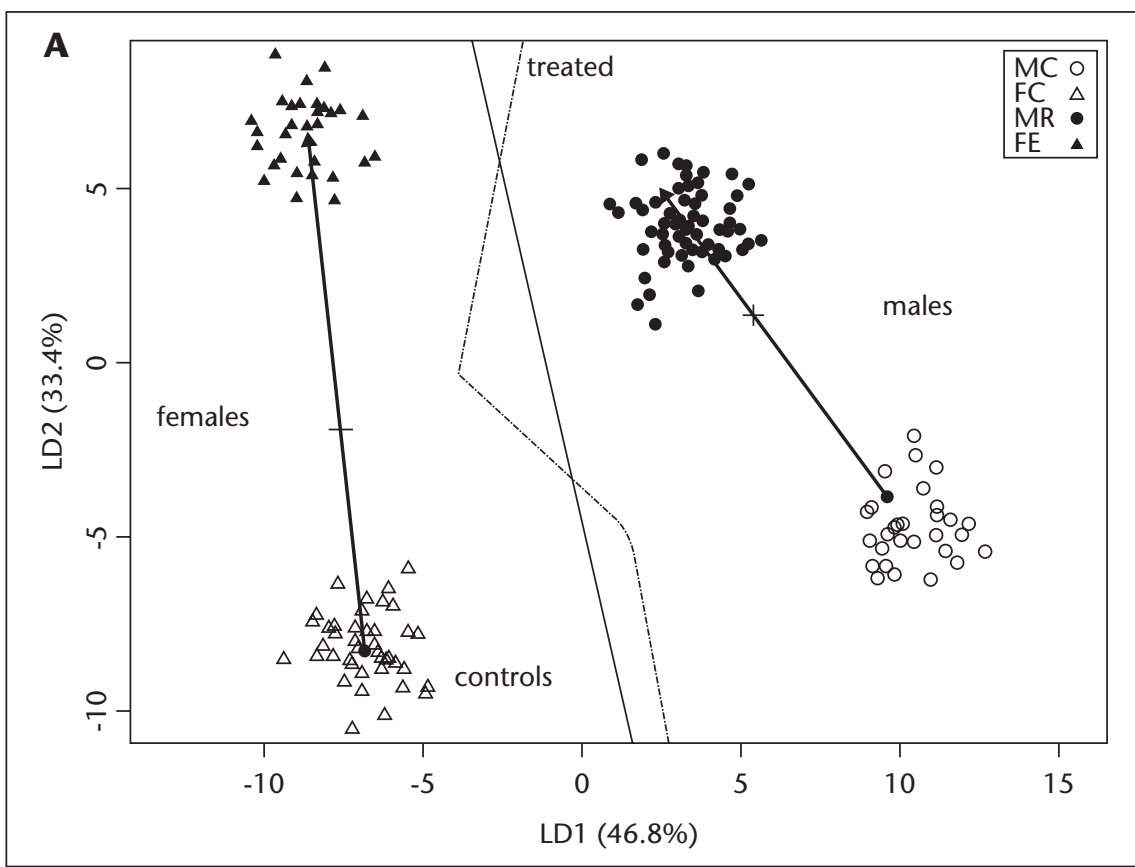

Figure 1. Représentation des disruptions hormonales apparaissant sur le premier plan factoriel de l'analyse factorielle discriminante réalisée sur 106 variables. Le premier axe factoriel discrimine les animaux suivant leur genre, le second résume la déviation métabolique commune subie par les animaux traités par des stéroïdes anabolisants, quel que soit leur genre. D'après Dumas et al. [10]. tions du métabolisme général pourront être interprétées en fonction des variations concomitantes du pattern endocrinien en cortisol, testostérone et IGF-1, ce dernier, un peptide, reflétant bien les variations de la stimulation somatotrope au niveau hépatique. Néanmoins, ces modélisations statistiques dont l'étude est en cours ne permettent pas pour l'instant de prédire avec certitude l'appartenance à telle ou telle classe de disruption endocrinienne expliquée par la prise de substances dopantes même si les analyses en cours permettent déjà de faire apparaître des ensembles de biomarquage métabolique relativement indépendants les uns des autres, dont nous pouvons espérer qu'ils pourront servir à reconstruire dans un terme proche des signatures métaboliques complexes témoignant de l'utilisation de cocktails de substances appartenant à différentes classes d'effets. II faut signaler les difficultés importantes que rencontre l'analyse des données métabonomiques, tant du point de vue statistique pour modéliser le fonctionnement des réseaux métaboliques en y intégrant la dimension physiologique que du point de vue de la description analytique desdits réseaux, bon nombre de variables statistiques n'étant pas encore suffisamment caractérisées au plan de la chimie analytique pour leur donner un sens biochimique. A ce titre, il faut signaler les efforts importants développés en matière d'analyse structurale réalisée par spectrométrie de masse et d'une manière moindre par RMN pour tenter de caractériser tous les biomarqueurs métaboliques d'intérêt et les réunir dans des bases de données accessibles à la communauté des chercheurs impliqués sur ces thématiques.

\section{Les phtalates : environnement et chaîne alimentaire}

Ces composés sont largement utilisés en plasturgie. Ils permettent de renforcer la souplesse de matières plastiques. Ils ne sont pas biologiquement neutres car ce sont des inducteurs de peroxysomes réputés puissants chez les espèces murines. De fait, une réévaluation complète de leur spectre "d'altération fonctionnelle » est en cours afin de mieux déterminer la dose sans effet pour l'homme car ils constituent une classe importante de contaminants alimentaires auxquels le consommateur est constamment exposé. Des études visant à documenter leurs propriétés de disruption endocrinienne indiquent que des effets peuvent être mesurés au niveau testiculaire. Ils sont répertoriés comme de possibles antiandrogènes et auraient de surcroît des propriétés œstrogéno-mimétiques. 
Une étude que nous avons réalisée a permis de montrer une variation de la réponse métabolique chez la souris liée à la quantité de diéthylhexyl-phtalate (DEHP) administré oralement pendant deux semaines [11]. L'effet hypolipémiant de cette molécule, largement décrit dans la littérature depuis de nombreuses années, se retrouve dans l'analyse métabolomique des empreintes plasmatiques. Dès la plus faible dose de $30 \mathrm{mg} / \mathrm{kg} / \mathrm{j}$ utilisée pour gaver les animaux, qui était réputée jusqu'à présent sans effet, une déviation significative des patterns métaboliques urinaire et hépatique est perceptible chez ces animaux, qui illustre bien le pouvoir résolutif de la méthode. Une modification du métabolisme énergétique se dégage de l'interprétation de la diminution relative de la proportion des acides aminés cétoformateurs, de l'augmentation parallèle de la proportion des acides aminés glucoformateurs et, enfin, de la baisse relative de la concentration en acides biliaires dans le cas d'une intoxication aiguë réalisée par la plus forte dose étudiée.

Une approche originale dans ce travail a consisté à rapprocher les données d'empreintes métaboliques et les données d'analyse de l'expression des gènes (transcriptome) obtenues sur les mêmes animaux. Cette contrainte supplémentaire a permis de produire une information encore plus structurée et de rapprocher ces deux types de données pour tenter de révéler des ensembles de biomarquage inédits. Par exemple, il est possible de faire apparaître un ensemble de régulations coordonnées qui est impliqué dans l'augmentation de la synthèse d'hème en cas d'intoxication par une forte dose de DEHP et qui s'expliquerait par une augmentation du transport de la thréonine dans les cellules, une augmentation de l'expression du gène Vanin1 impliqué dans la biosynthèse de l'acide pantothénique et de la cystéamine et une augmentation de l'expression du gène Alas1 impliqué dans la biosynthèse, à partir de la glycine, de l'acide 5-aminolévulinique, un précurseur de l'hème [11].

Ainsi, cette étude a permis de découpler les variations du métabolisme reflétant celles physiologiques, vraisemblablement réversibles, de celles, plus fortes, attestant un réel impact toxique. Ce travail, à titre d'exemple, souligne bien l'importance de réaliser des approches combinées, ici métabonomique et transcriptomique, pour mettre en exergue des biomarqueurs dont l'interprétation duale révèle, quand on sait les interpréter, la richesse des mécanismes biochimiques sous-jacents à l'œuvre dans les phénomènes d'ajustement homéostatique qui prennent place en cas de perturbation toxique. À défaut, cette démarche permet néanmoins de révéler l'existence de biomarqueurs incon- nus qu'il s'agira de caractériser en recourant à des méthodes biochimiques plus conventionnelles.

\section{Toxi-expositions et disruptions métaboliques : le cas de la porcelle enracinée}

La versatilité de l'approche métabonomique a pu être pleinement illustrée dans le cas d'une recherche de métabolites secondaires présents chez la Porcelle enracinée (Hypochœris radicata L.), certains étant toxiques pour l'espèce équine, en essayant de valider le modèle souris comme modèle de disruptions métaboliques orthologue de celles présentes chez le cheval intoxiqué, plus précisément en ciblant les tissus cibles de l'intoxication, et en essayant dans le même temps de caractériser les stades végétatifs comprenant les fractions d'extraits végétaux les plus toxiques. L'ingestion de cette plante est à l'origine de troubles neuromusculaires graves dans l'espèce équine (harper australien ou Australian Stringhalt) ; aucune thérapie n'existe pour l'instant. Bien que cette maladie soit connue depuis 150 ans, l'étiologie et les mécanismes physiopathologiques sont pour l'instant ignorés. Cette recherche a pu être initiée sans qu'aucune connaissance sur un quelconque mécanisme d'intoxication ou une quelconque famille de substances toxiques n'ait été a priori nécessaire pour conduire cette recherche [12]. Un biomarqueur métabolique candidat a été retrouvé : le scyllo-inositol. II est présent à de très faibles concentrations chez les animaux témoins mais est retrouvé en fortes concentrations dans le foie et le cerveau des souris (figure 2) ou des rats intoxiqués pendant deux ou trois semaines par de faibles concentrations (1-3\%) de poudre de fleur incorporée dans I'aliment.

Une augmentation de ce marqueur a déjà été décrite dans d'autres troubles neurologiques comme l'alcoolisme chronique [13] ou la maladie d'Alzheimer [14]. Le scyllo-inositol est présent physiologiquement à de très faibles concentrations au sein des systèmes nerveux périphérique et central mais ses fonctions précises sont encore peu connues. Le scylloinositol et son stéréoisomère, le myo-inositol, sont reliés à l'activité de pompes à sodium et, de ce fait, sont impliqués dans les processus de conduction nerveuse au sein des nerfs périphériques, lesquels peuvent être altérés lors de neuropathie diabétique périphérique accompagnée d'axonopathie distale [15]. De plus, des modifications de la concentration des inositols ont été recensées lors de désordres comportementaux comme lors de dépression [16]. L'implication du scyllo-inositol chez les animaux consommant Hypochœris radicata atteste de disruptions évidentes affectant de façon conjointe les neurones et les cellules gliales. Les analogies dans les perturbations métaboliques

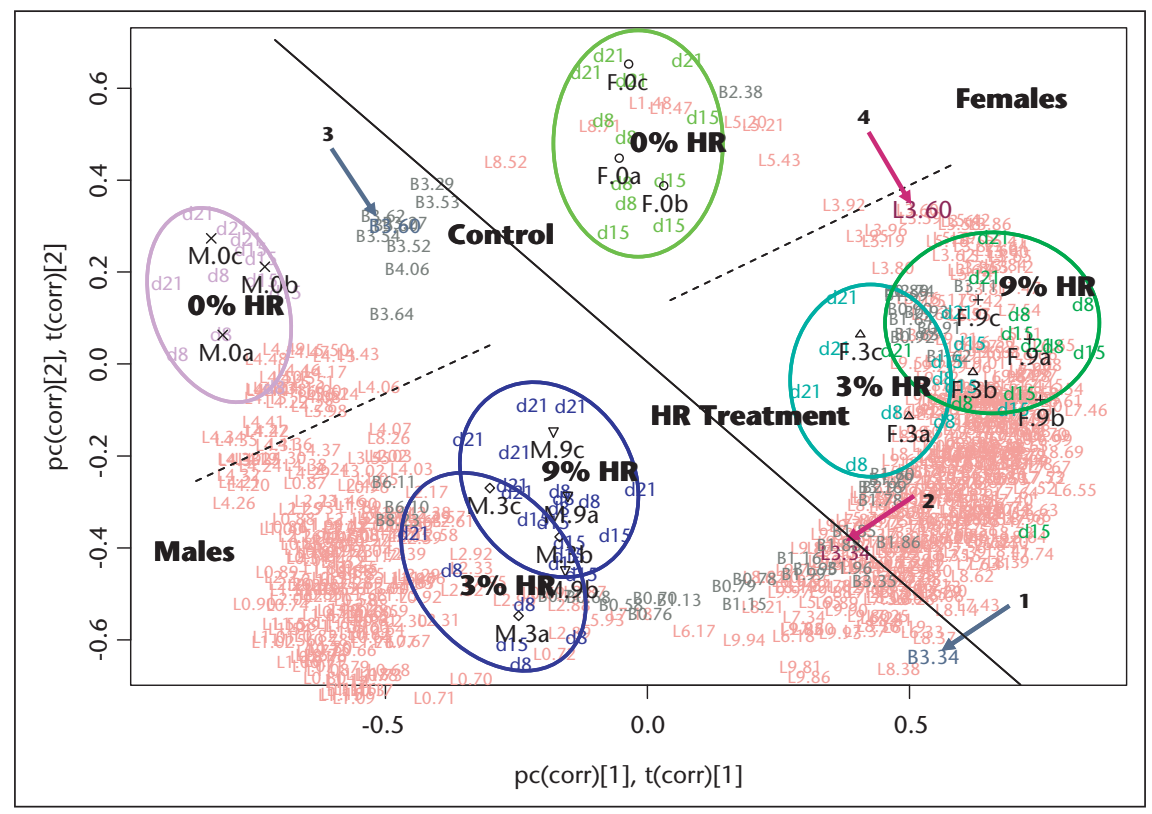

Figure 2. Biplot obtenu à partir des 2 premières composantes PLS résumant les variables métabolomiques représentant la fraction hydrosoluble extraite du cerveau et celle extraite du foie. La projection des variables ayant une norme supérieure à 0,5 apparait en gris pour le cerveau et en rouge pour le foie. La variable intitulée B3.34 (flèche $n^{\circ} 1$ ) correspond au scyllo-inositol détecté à $\delta=3,34$ ppm dans le cerveau et la même variable hépatique est intitulée L3.34 (flèche $n^{\circ} 2$ ). Les variables B3.60 (flèche $n^{\circ} 3$ ) et L3.60 (flèche $n^{\circ} 4$ ) correspondent au myo-inositol détecté à $\delta=3.60$ ppm respectivement dans le cerveau et le foie. HR : Hypochoeris radicata. D'après Domange et al. [17]. 
et les manifestations physiopathologiques entre maladies humaines et le harper australien permettent de proposer des hypothèses concernant la pathogénie de ce trouble.

Une validation de la démarche de recherche conduite en métabonomique a pu être produite par des analyses fonctionnelles par imagerie cérébrale obtenue par résonance magnétique nucléaire sur animal vigile [17] ou au moyen d'études approfondies du comportement. La confrontation des données obtenues par RMN in vitro et par imagerie in vivo a permis de recenser un ensemble de métabolites potentiellement impliqués dans les perturbations métaboliques après ingestion d'Hypochœris radicata. Parmi ceux-ci, I'augmentation de choline serait en lien avec des processus d'inflammation et de démyélinisation, fait également conforté par l'augmentation de $\mathrm{N}$-acétyl-aspartate, assez rare, mais retrouvée également dans le syndrome de Cavanan [18, 19]. Les phénomènes d'hypoxie et l'état de stress oxydatif entraîneraient une augmentation de lactate et d'inosine. Enfin, le déséquilibre de la balance glutamine/glutamate expliquerait l'excitotoxicité et les perturbations de conduction neuronale. Les modifications comportementales observées chez la souris, dont la diminution de l'état d'anxiété dans un contexte sécurisé et l'augmentation de l'activité motrice, sembleraient être le signe de perturbations des processus de neurotransmission et d'excitotoxicité, impliquant le myo-inositol, le $\mathrm{N}$-acétyl-aspartate [20] et la teneur en calcium intracellulaire.

L'utilisation conjointe de ces trois méthodes d'exploration, la métabonomique, l'imagerie cérébrale et l'étude comportementale, a permis, en se fondant sur le principe d'orthologie, de dresser un réseau de voies métaboliques pouvant être affectées par l'intoxication consécutive à l'ingestion d'Hypochœris radicata.

\section{Conclusion}

Les recherches conduites dans le domaine de la métabonomique sont en plein essor [21]. Elles profitent pour beaucoup des avancées réalisées en métabolomique, le support analytique incontournable de l'analyse dynamique des variations du métabolisme, qui a progressé très sensiblement ces toutes dernières années grâce aux progrès très significatifs obtenus en ce qui concerne la résolution des spectromètres de masse disposant de détecteurs de type temps de vol, orbitrap ou résonance cyclotronique des ions ou la sensibilité et, dans une moindre mesure, la résolution des spectromètres de résonance magnétique nucléaire. À ce développement instrumental sans précédent se conjuguent les avancées des recherches en statistique et en bioinformatique qui permettent désormais de construire des recherches plus ciblées visant à valider tel ou tel modèle d'analyse des perturbations des systèmes biologiques, en particulier quand se pose encore la question de la pertinence d'une analyse des possibles effets engendrés par les apports chroniques via l'alimentation des faibles doses de xénobiotiques, et ce durant de longues périodes.

La versatilité de l'approche métabonomique permet en outre de conduire des recherches visant à qualifier l'innocuité des systèmes alimentaires complexes qui incorporent par exemple, comme matières premières, des ingrédients préparés à partir de plantes génétiquement modifiées (PGM) ou bien, comme compléments alimentaires, des extraits de plantes douées de certaines propriétés médicinales. Dans l'un ou l'autre des cas, les méthodes d'expertise qui dérivent pour la majeure partie d'entre elles des méthodes utilisées en recherche pharmaceutique ne sont guère appropriées car elles reposent sur l'existence d'un facteur de sécurité, concept qui est inopérant dans le cas des matières premières alimentaires, ou bien elles visent à établir les propriétés d'une seule molécule à visée thérapeutique, concept pris en défaut dans le cas d'extraits complexes de plantes.

Des premiers travaux conduits par notre équipe sur le thème des PGM ont ainsi permis de mieux caractériser l'impact des facteurs génétiques (variétal et transgénique) et des facteurs agronomiques sur le phénotype métabolique de tubercules de pomme de terre examiné par spectrométrie de masse (Py-MAB-ToF MS) [22]. Bien que mesurables, les effets imputables aux gènes insérés dans les différents cultivars étudiés, qu'ils procurent une protection au virus $Y$ de la pomme de terre ou une capacité de métabolisation des nitrates, ont un impact modeste sur le phénotype métabolique des tubercules, les facteurs liés aux conditions de culture (traitement pesticide) et au cultivar ayant un impact beaucoup plus important. Une exploration métabonomique de la qualité alimentaire de ces différents cultivars réalisée chez le rat a permis là aussi de hiérarchiser l'importance de certains de ces facteurs, le facteur cultivar ayant un effet majeur comparativement à celui de l'événement génétique étudié.

Pour résumer, la métabonomique qui allie analyse métabolomique et analyse statistique multidimensionnelle s'avère donc être un outil efficace et précieux pour mieux définir des métriques caractérisant les perturbations d'origine toxique des systèmes vivants, quelle qu'en soit l'intensité, ces métriques pouvant à l'avenir aider à mieux renforcer l'expertise toxicologique des substances xénobiotiques retrouvées dans les aliments.

\section{RÉFÉRENCES}

1. Nicholson JK, Lindon JC, Holmes E. 'Metabonomics' : understanding the metabolic responses of living systems to pathophysiological stimuli via multivariate statistical analysis of biological NMR spectroscopic data. Xenobiotica $1999 ; 29$ : 1181-9.

2. Holmes E, Foxall P], Nicholson JK, et al. Automatic data reduction and pattern recognition methods for analysis of $1 \mathrm{H}$ nuclear magnetic resonance spectra of human urine from normal and pathological states. Anal Biochem 1994 ; 220 : 284-96.

3. Holmes E, Nicholls AW, Lindon JC, et al. Development of a model for classification of toxininduced lesions using $1 \mathrm{H}$ NMR spectroscopy of urine combined with pattern recognition. NMR Biomed 1998 ; 11 : 235-44.

4. Coen M, Hong YS, Clayton TA, et al. The mechanism of galactosamine toxicity revisited; a metabonomic study. I Proteome Res $2007 ; 6$ : 2711-9.

5. Yap IK, Clayton TA, Tang $\mathrm{H}$, et al. An integrated metabonomic approach to describe temporal metabolic disregulation induced in the rat by the model hepatotoxin allyl formate. J Proteome Res $2006 ; 5$ : 2675-84.

6. Lindon JC, Keun HC, Ebbels TM, Pearce JM, Holmes E, Nicholson JK. The Consortium for Metabonomic Toxicology (COMET) : aims, activities and achievements. Pharmacogenomics $2005 ; 6$ : 691-9.

7. Lindon JC, Nicholson JK, Holmes E, et al. Contemporary issues in toxicology the role of metabonomics in toxicology and its evaluation by the COMET project. Toxicol Appl Pharmacol $2003 ; 187$ : 137-46.

8. Ebbels TM, Keun HC, Beckonert OP, et al. Prediction and classification of drug toxicity using probabilistic modeling of temporal metabolic data : the consortium on metabonomic toxicology screening approach. J Proteome Res 2007 ; $6: 4407-22$.

9. Dumas ME, Canlet $\mathrm{C}$, André $\mathrm{F}$, Vercauteren I, Paris A. Metabonomic assessment of physiological disruptions using ${ }^{1} \mathrm{H}^{13} \mathrm{C}$ HMBC-NMR spectroscopy combined with pattern recognition procedures performed on filtered variables. Anal Chem 2002 ; 74 : 2261-73.

10. Dumas ME, Canlet C, Vercauteren J, André F, Paris A. Homeostatic signature of anabolic steroids in cattle using ${ }^{1} \mathrm{H}^{13} \mathrm{C} \mathrm{HMBC}$ NMR metabonomics. J Proteome Res 2005 ; 4 : 1493-502.

11. Eveillard $A$, Lasserre $F$, de Tayrac $M$, et al. Potential mechanisms of toxicity after di-(2ethylhexyl)-phthalate (DEHP) adult exposure in the liver and testis using a systems biology approach. Toxicol Sci 2009 ; (Soumis).

12. Domange C. Etude des disruptions métaboliques chez le rongeur suite à l'ingestion d'Hypochœeris radicata (L.), plante toxique pour 
l'espèce équine. Validation de l'approche métabonomique par des études comportementales et par imagerie cérébrale. Thèse de doctorat de l'Université de Toulouse. 5 novembre 2008.

13. Viola A, Nicoli F, Denis B, et al. High cerebral scyllo-inositol : a new marker of brain metabolism disturbances induced by chronic alcoholism. MAGMA $2004 ; 17$ : 47-61.

14. Griffith HR, den Hollander JA, Stewart CC, et al. Elevated brain scyllo-inositol concentrations in patients with Alzheimer's disease. NMR Biomed $2007 ; 20$ : 709-16

15. Hirano F, Tanaka H, Okamoto K, et al. Natural course of diabetic peripheral neuropathy in spontaneous-onset diabetic Chinese hamsters. Diabetes Res Clin Pract 1995 ; 28 : 151-9.
16. Barkai Al, Dunner DL, Gross HA, Mayo P, Fieve RR. Reduced myo-inositol levels in cerebrospinal fluid from patients with affective disorder. Biol Psychiatry $1978 ; 13: 65-72$.

17. Domange $C$, Canlet $C$, Traorè A, et al. Orthologous metabonomic qualification of a rodent model combined with magnetic resonance imaging for an integrated evaluation of the toxicity of Hypochoeris radicata. Chem Res Toxicol $2008 ; 21: 2082-96$.

18. Austin SJ, Connelly A, Gadian DG, Benton JS, Brett EM. Localized ${ }^{1} \mathrm{H}$ NMR spectroscopy in Canavan's disease : a report of two cases. Magn Reson Med 1991 ; 19 : 439-45.

19. Grodd W, Krageloh-Mann I, Petersen D, Tre$\mathrm{fz}$ FK, Harzer K. In vivo assessment of $\mathrm{N}$-acetylaspartate in brain in spongy degeneration (Canavan's disease) by proton spectroscopy. Lancet $1990 ; 336: 437-8$.
20. Kitada K, Akimitsu T, Shigematsu Y. Accumulation of $\mathrm{N}$-acetyl-L-aspartate in the brain of the tremor rat, a mutant exhibiting absence-like seizure and spongiform degeneration in the central nervous system. I Neurochem 2000 ; $74: 2512-9$

21. Coen M, Holmes E, Lindon JC, Nicholson JK. NMR-based metabolic profiling and metabonomic approaches to problems in molecular toxicology. Chem Res Toxicol 2008 ; 21 : 9-27.

22. Priymenko N, Canlet $\mathrm{C}$, Gottardi $\mathrm{G}$, et al. Etude métabonomique en RMN du $1 \mathrm{H}$ de l'adaptation de rats en croissance à un régime préparé à partir de pommes de terre génétiquement modifiées. In : 2e journées scientifiques du Réseau Français de Métabonomique et Fluxomique. Saint-Sauves d'Auvergne, 13-15 décembre 2006. 